\title{
La paloma y otras aves como reservorio de Cryptococcus spp.
}

\author{
Inmaculada Rosario ${ }^{1}$, Begoña Acosta ${ }^{1}$, Francisca Colom ${ }^{2}$

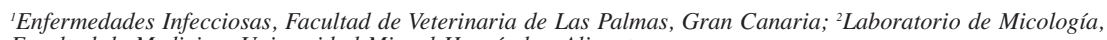 \\ Facultad de Medicina, Universidad Miguel Hernández, Alicante \\ Resumen En los últimos 25 años, los casos de criptococosis humana y animal han \\ aumentado considerablemente, en gran medida debido a la supervivencia de \\ enfermos con alteraciones en el sistema inmunológico. En numerosas \\ situaciones, la enfermedad se ha relacionado con la exposición de los \\ pacientes a excreciones de aves. De ellas, la paloma urbana-Columba livia- \\ es sin duda la más importante como reservorio de la levadura, pero el estudio \\ de una gran diversidad de especies de pájaros, deja claro que no es la única \\ portadora de criptococos patógenos. La sospecha de que estas aves sean la \\ fuente de la enfermedad está comenzando a ser demostrada por la aplicación \\ de métodos moleculares de tipificación que permiten comparar, con un alto \\ grado de discriminación, las cepas encontradas en pacientes y en los \\ animales de su entorno más próximo. \\ Palabras clave Cryptococcus spp., Cryptococcus neoformans, Paloma, Aves, Epidemiología

\section{Pigeons and other birds as a reservoir for Cryptococcus spp.} \\ Summary In the last 25 years, the cases of human and animal cryptococcosis have \\ increased significantly. This is mostly due to the improvement in the survival of \\ immunocompromised patients. The disease is frequently related to the \\ exposure of this type of patients to avian droppings. Among birds, pigeon \\ -Columba livia- is undoubtedly the most important reservoir for the \\ Cryptococcus species. Nevertheless, the study of a large number of bird's \\ species demonstrated that pigeons are not the only Cryptococcus spp. \\ carriers. The suspicion of the birds being the source for the infection is now \\ becoming a demonstrable fact thanks to the use of molecular typing methods. \\ These methods allow the comparison between strains from birds to patients \\ living around them, with high level of discrimination.
}

Key words C Cryptococcus spp., Cryptococcus neoformans, Pigeon, Birds, Epidemiology

\section{Introducción}

Dentro del género Cryptoccoccus se han aislado y descrito más de 38 especies. El denominado complejo Cryptococcus neoformans, incluye las dos especies patógenas más importantes de este género, Cryptococcus neoformans y Cryptococcus gattii, que anteriormente se consideraban variedades de una sola especie: $C$. neoformans var. neoformans y C. neoformans var. gattii [33]. Estas le- vaduras presentan, entre otras, importantes diferencias en su ecología y en la epidemiología de la criptococosis que producen. $C$. neoformans se aísla en todo el mundo, mostrando preferencia por las regiones templadas y los suelos contaminados con heces de aves (por tanto, con alta concentración de creatinina); $C$. gattii se asocia a regiones tropicales y subtropicales y se aísla de distintas especies de eucaliptos. Otras especies del género también han sido aisladas de heces de aves, suelos y vegetales [30,35,47,65]. La falta de evidencias de una transmisión directa individuo-individuo, sustenta la hipótesis de que la infección debe adquirirse de estas fuentes medioambientales por inhalación $[7,51]$.

\section{La paloma y Cryptococcus neoformans}

El papel de la paloma como portadora de hongos patógenos fue establecido por Emmons [13] en 1955, el cual aisló $C$. neoformans de las excreciones de palomas urbanas (Columba livia), siendo el primero en establecer la relación existente, y actualmente consolidada, entre el microorganismo y las heces de estas aves. Estudios posteriores realizados por el propio Emmons [14] y por otros
Dirección para correspondencia:

Dra. Begoña Acosta Hernández

Carretera de Trasmontaña, $s / n$

35416 Arucas, Gran Canaria, España

Tel.: + 34928454360

E-mail: bacosta@dpat.ulpgc.es

(ㄷ)2008 Revista Iberoamericana de Micología

Apdo. 699, E-48080 Bilbao (Spain)

$1130-1406 / 01 / 10.00 €$ 
investigadores muestran que las excreciones viejas de palomas son la fuente conocida más abundante de dicho microorganismo. Tras el descubrimiento de Emmons, investigadores de casi todo el mundo han demostrado que las deposiciones de paloma son un importante reservorio de C. neoformans (Tabla 1). Según algunos autores, este hongo no suele aislarse en deyecciones recientes [29,42], pero sí en las acumuladas y secas existentes en palomares, aleros de edificios, áticos o balcones de casas abandonadas donde duermen las palomas. Este hábitat desecado, alcalino, rico en sales y nitrógeno, es ecológicamente restrictivo pero no es infrecuente en el medio ambiente urbano [49,56]. Otros estudios no muestran diferencias significativas en la frecuencia de aislamiento entre los excrementos secos y los frescos [8], o incluso aíslan el microorganismo con más facilidad de heces frescas [9]. Parece que la alta concentración de creatinina en el estiércol de paloma favorece el crecimiento de los criptococos, pero, además, las heces de pichón, brindan otras características: ambiente alcalino, hiperosmolar y rico en muchos compuestos nitrogenados, además de la creatinina [43]. Las concentraciones de esta levadura en el excremento de paloma a menudo exceden $10^{6}$ organismos viables por gramo, su alta concentración en este substrato puede estar también relacionada con su habilidad para asimilar no sólo la creatinina, sino también la xantina, la urea y el ácido úrico, compuestos abundantes en los excrementos de las aves [57]. Aún así, $C$. neoformans puede desaparecer cuando los detritos de estas aves se mezclan con el suelo [12]. De hecho, pocas veces se aísla de suelos orgánicamente enriquecidos [14].

Se ha estimado que la permanencia de la levadura en deyecciones de palomas a la sombra, húmedas o desecadas, puede ser de hasta más de dos años. Aunque hasta hace poco se consideraba que la exposición directa al sol destruía el hongo o inhibía su crecimiento [7,12,56], actualmente parece que la capacidad de las especies patógenas de Cryptococcus para producir pigmentos melanoides no sólo les permite sobrevivir a la radiación solar, sino que pueden llegar a utilizar las radiaciones como energía metabólica [36]. Este hecho, posiblemente, les permite sobrevivir hasta que los excrementos se convierten en polvo. El polvo vehicula levaduras de solo 1-2 micras de diámetro y acapsuladas, lo que les permite alcanzar fácilmente el espacio alveolar al ser inhaladas [7,17,42].

Además de las características propias de las heces de las aves, otros factores pueden determinar la probabilidad de encontrar a esta levadura en las excreciones de palomas. De hecho, el sistema de anidación resulta crucial para el aislamiento de $C$. neoformans de estas muestras, resultando mayor cuando se acumulan excrementos de más de 15 palomas en un mismo lugar [6], con lo que se subraya la importancia de evitar la acumulación de deyecciones de palomas, ya que si el grado de contaminación ambiental por $C$. neoformans fuese elevado, este podría convertirse en un peligro para la salud pública, y más concretamente para las personas dedicadas al cuidado, cría y limpieza de las palomas y palomares (Figura 1), así como para aquellos que han de remover la tierra contaminada con heces de paloma y los que alimentan a palomas salvajes o en libertad $[19,21,26,58]$.

Dada la importancia que en salud pública tiene el conocer el reservorio de un microorganismo patógeno, la posible presencia y permanencia de $C$. neoformans en el tubo digestivo de las palomas es un tema que despierta gran interés. En 1976, Swinne-Desgain [64] realizó un estudio con 10 palomas a las que suministró células levaduriformes vía oral. Previamente había comprobado que los animales estaban libres del microorganismo en buche y deyecciones. Pudo observar que un día después de la ino- culación, la levadura se recuperaba de las heces de nueve de las 10 palomas, y que a los 22 días de la ingestión todavía existía una muestra positiva. Asimismo, observó que las muestras de buche eran positivas durante un período de tiempo más largo, ya que dos de las palomas todavía presentaban el microorganismo en el día 86 post-inoculación. Estos resultados y otros obtenidos previamente [63] indican que la paloma puede portar $C$. neoformans en su buche y que la levadura puede sobrevivir en él y, ocasionalmente, pasar a heces, donde se multiplica. Las palomas con $C$. neoformans en su tracto intestinal pueden excretar el organismo durante varios meses sin desarrollar la enfermedad [52].

El aislamiento de C. neoformans del buche de paloma ha sido descrito en múltiples ocasiones [4,5,23,54,55,64], así como su persistencia en otras localizaciones del cuerpo de las aves (cloaca, plumas, patas, pico). Sin embargo, es frecuente que los autores concluyan que, si bien la paloma es portadora de $C$. neoformans, estas aves no deben considerarse reservorios de la misma [27]. Littman y Borok [39] demostraron que la concentración de criptococos en el pico, buche, patas y muestras de cloaca de estas aves es baja, y afirman que es poco probable que la paloma en sí sea la principal fuente de criptococos en la Naturaleza. Abou-Gabal y Atia [1] confirman que la paloma no juega un papel biológico activo en la diseminación de $C$. neoformans en la naturaleza porque no favorece la multiplicación del hongo en su buche. Estos autores observaron el efecto inhibitorio sobre el crecimiento de $C$. neoformans, de bacterias presentes en el contenido intestinal de palomas sanas (Pseudomonas aeruginosa, Bacillus subtilis, Staphylococcus albus, Streptococcus faecalis, Escherichia coli, Proteus mirabilis y Klebsiella aerogenes).

\section{Otras aves relacionadas con los criptococos patógenos}

Como hemos visto, el hábitat principal de $C$. neoformans es el suelo contaminado con deyecciones de paloma, aunque también se ha aislado de nidos de otros pájaros y de excreciones de otras aves. En la tabla 1 se recogen un buen número de estudios realizados en este sentido con muy diversas especies de aves. Los resultados obtenidos en estos trabajos no son homogéneos. Muchos de ellos muestran el aislamiento de $C$. neoformans y otros criptococos, tanto de muestras de excreciones como de buche o cloaca de las aves, mientras que en otros, a veces realizados con las mismas especies, apenas si se detecta la levadura. Staib y Schulz [61] analizaron excrementos procedentes de 142 especies de aves consiguiendo el aislamiento de C. neoformans tan sólo en cuatro de todas las especies estudiadas: Probosciger aterrimus (cacatúa de las palmas), Ara militaris (guacamaya verde), Psittacus erithacus (loro gris africano) y Podargus strigoides (podargo castaño). Mancianti et al. [40] analizaron 325 muestras de heces desecadas y frescas de aves pertenecientes a la familia de las psitácidas, no obteniendo $C$. neoformans en ninguna de ellas. Esto sugiere que deben existir factores independientes de la propia composición de las heces o de la temperatura y condición del tubo digestivo de las aves, que influyen decisivamente en la presencia y persistencia de la levadura en este entorno.

\section{Aves y criptococosis}

La adquisición de criptococosis a partir de aves o substratos procedentes de aves se ha sospechado en numerosas ocasiones $[7,10,15,18,30,44,59,65,67,69,70]$. El primer caso de criptococosis humana directamente atribuido 
Tabla 1. Especies de aves y aislamientos de Cryptococcus relacionados.

\begin{tabular}{|c|c|c|c|}
\hline Nombre común & Especie & $\begin{array}{l}\text { Especies de Cryptococcus } \\
\text { encontradas }\end{array}$ & Referencias \\
\hline Paloma urbana & Columba livia & C.n., C.a., C.I., C.t., C.u. & $\begin{array}{l}2,9,16,20,21,22,23,28,29,30 \\
38,42,46,53,54,55,62,71,72\end{array}$ \\
\hline Tórtolas & Zenaida auriculata & C.n., C.a. & 30,59 \\
\hline Gallina & Gallus gallus & C.n., C.a.C. I. & $30,37,59,65,41$ \\
\hline Gallina de Guinea & n.e. (familia Numididae) & C. $n$. & 65 \\
\hline Patos & n.e. (familia Anatidae) & C. $n$. & 65 \\
\hline Gansos & Chloephaga spp. & C. $n$. & 65 \\
\hline Avefría & Vanellus vanellus & C. $n$. & 65 \\
\hline Urraca común & Pica pica & C. $n$. & 34 \\
\hline Cernícalo vulgar & Falco tinnunculus & C. $n$. & $5^{*}$ \\
\hline Cernícalo primilla & Falco naumanni & C. a. & 5 \\
\hline Halcón peregrino & Falco subuteo & C. I. & 5 \\
\hline Bucardo (águila ratonera) & Buteo buteo & C. $n$. & $5^{*}$ \\
\hline Milano negro & Milvus nigrans & C. $n$. & 5 \\
\hline Cerceta común & Anas crecca & C. a., C. I. & $6^{*}$ \\
\hline Focha común & Fulica atra & C. a., C. I. & $6^{*}$ \\
\hline Chocha perdiz & Scolopax rusticola & C. a. & $6^{*}$ \\
\hline Gorrión & Passer domesticus & C. n., C. a. & $30,37,59,65$ \\
\hline Estornino & Sturnus vulgaris & C. n., C. a. & $30,37,59,65$ \\
\hline Alondra & Alauda arvensis & C. n., C. a. & $30,37,59$ \\
\hline Golondrina & n.e. (familia Hirundinidae) & C. $n$. & 65 \\
\hline Cardenal común & Paroaria dominicana & C. $n$. & 50 \\
\hline Canario & Serinus canarius & C. n., C. a. & $11,30,37,50,59,60,65,69$ \\
\hline Jilguero dorado & Sicalis flaveola & C. $n$. & 50 \\
\hline Corbatita común & Sporophila caerulescens & C. $n$. & 50 \\
\hline Munia & Passeriformes (orden) & C. $n$. & 48 \\
\hline Loro & n.e.(familia Psitacidae) & C. n., C. a., C. l. & $11,30,41,50,60,61,67,69$ \\
\hline Guacamayo verde & Ara militaris & C. $n$. & 61 \\
\hline Martin pescador & & C. I. & 40 \\
\hline Periquito & n.e. (familia Psitacidae) & C. n., C. a. & $30,37,59$ \\
\hline Cacatúa & Cacatua alba & C. $n$. & 45 \\
\hline Cacatúa & Probosciger aterrimus & C. $n$. & 61 \\
\hline Ninfa & Nynphicus hollandicus & C. $n$. & 34 \\
\hline Podargo castaño & Podargus strigoides & C. $n$. & 61 \\
\hline $\begin{array}{l}\text { Heces de aves sin } \\
\text { especificar especies }\end{array}$ & $\begin{array}{l}\text { n.e. Aviarios con } \\
\text { distintas especies }\end{array}$ & $\begin{array}{l}\text { C. n., C. I., C. u., C. t., C. gastricus, } \\
\text { C. luteolus, C. adeliensis }\end{array}$ & $31,66,68$ \\
\hline
\end{tabular}

al contacto con excreciones de palomas fue documentado por Littman en el año 1959 [cit. 39]. Passoni et al. [50], en un amplio estudio realizado en Brasil en el entorno doméstico de enfermos de sida, encuentran una marcada relación entre la presencia de la levadura en polvo doméstico de las viviendas donde hay aves de compañía $(25 \%)$ con respecto a las que no tienen estos animales $(9,3 \%)$. Pero, realmente, la transmisión de la enfermedad desde diferentes tipos de aves o sus substratos sólo ha podido demostrarse después del desarrollo de métodos de biología molecular para la tipificación intraespecífica de cepas. En este sentido, existen escasos pero interesantes trabajos en los que se demuestra que la cepa patógena responsable de la criptococosis está estrechamente relacionada o es idéntica a la saprofita en las heces o tubo digestivo de un ave de compañía. Nosanchuck et al. [45] encontraron el mismo biotipo y genotipo en la cepa que provocó criptococosis a una paciente trasplantada, que en la obtenida de la cacatúa (Umbrella cockatoo) que tenía en casa. Compararon perfiles bioquímicos, serotipo, electrocariotipo e hibridación (southern blot) con el fragmento CNRE1. Los aislamientos fueron idénticos. Más adelante, Bauters et al. [3] publica- ron el caso de un paciente traqueotomizado cuya prótesis de voz estaba colonizada por una cepa de $C$. neoformans que mediante tipificación bioquímica y molecular, fue indistinguible de la aislada de las heces de sus palomos deportivos. En este caso se obtuvo el perfil molecular mediante amplificación por PCR con la secuencia (GACA)4. Por último, Lagrou et al. [34] encontraron cepas genéticamente indistinguibles en las heces de una urraca (Pica pica) enjaulada y en las de la paciente que convivió unas semanas en la misma casa. En este caso se trataba de una enferma inmunocompetente y las cepas se estudiaron mediante electrocariotipificación y AFLP (Amplified Fragment Lenght Polymorphism), que es uno de los métodos moleculares con mayor poder discriminativo.

Además de estos casos, la posible transmisión desde aves a humanos se ha sospechado en otras ocasiones, aunque no ha podido demostrarse. En 1983, Wegener y Staib [69] publicaron el caso de una paciente de 69 años, aficionada a las aves, a la que se le diagnosticó postmortem una criptococosis que afectaba al sistema nervioso central; sugirieron que el material fecal de un periquito $(\mathrm{Me}$ lopsittacus undulatus) fuera la posible fuente de infección. 

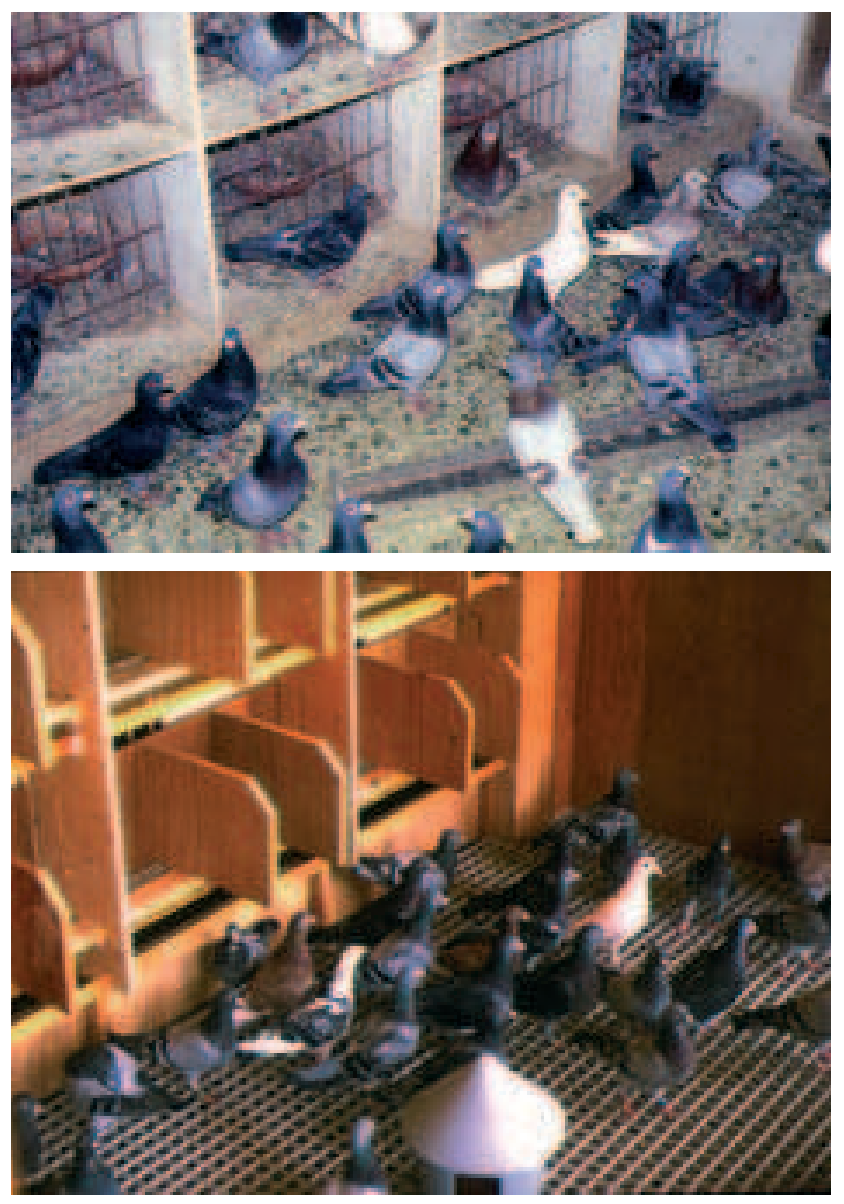

Figura 1. Dos situaciones higiénicas distintas en palomares.

Tokojima et al. [67] en el año 2001, describieron un caso de criptococosis en una señora aficionada a los pájaros y sugirieron el posible contagio a partir de las heces de un loro. En el primer caso de criptococosis por C. gatti descrito en España, tras un exhaustivo muestreo en el ambiente del paciente, se concluyó que la fuente del patógeno debió ser un ave tropical del vecindario, a la que no se pudo tener acceso [10].

\section{Otros criptococos en palomas y otras aves}

En los estudios realizados para la búsqueda de cepas del complejo C. neoformans, algunos autores describen la presencia de otras especies de Cryptococcus, con valoraciones diversas acerca de la importancia de este hallazgo. En la literatura revisada, las especies descritas incluyen Cryptococcus albidus, Cryptococcus laurentii, Cryptococcus uniguttulatus, Cryptococcus humiculus, Cryptococcus curvatus y Cryptococcus adeliensis (Tabla 1).
$C$. laurentii ha sido aislado de excrementos de diferentes especies aviares tales como lechuzas, martín pescador, loros, y gallinas enfermas y sanas [40]; este último trabajo llega a la conclusión de que el confinamiento de estas aves puede ser una potencial fuente de contagio para el personal relacionado con la industria avícola que presente alterado su sistema inmune. Además, otros trabajos documentan el aislamiento de esta especie en excrementos y cloacas de cerceta y focha [5], así como de palomas $[42,54,55]$. Rosario et al. consiguen también el aislamiento de esta especie de criptococo a partir de muestras de buche de palomas [54,55].

C. uniguttulatus ha podido ponerse de manifiesto en deyecciones y muestras de cloaca de paloma e, incluso, se ha conseguido también el aislamiento de esta especie a partir de las muestras de buche [42,54,55]. C. albidus ha sido aislado recientemente de muestras de cloaca, muestras de buche y de excrementos de palomas [22,24,54,55]. También ha podido ser aislado de excrementos o nidos de otras aves (Tabla 1).

La mayoría de estos hallazgos son aportaciones no relacionadas con el desarrollo de enfermedad humana o animal pero, en algunos casos, $C$. albidus y $C$. laurentii han sido descritos como patógenos $[25,32] \mathrm{y}$, recientemente, una cepa de $C$. adeliensis aislada de excrementos de aves, se ha descrito como el agente causal de un caso de criptococosis en Alemania [66].

\section{Conclusiones}

Parece sobradamente demostrado que hay una estrecha relación entre la paloma y la especie C. neoformans y que esta es la portadora por excelencia de la levadura. No obstante, el hongo ha podido aislarse de otras muchas especies de aves. En cualquier caso, la mera presencia de las aves no debe considerarse un riesgo sanitario. Cryptococcus neoformans es un saprofito del tubo digestivo de un buen número de especies aviarias, pero parece que son los excrementos de estos animales los que constituyen un hábitat importante para la levadura patógena. La elevada capacidad de supervivencia del microorganismo en estos sustratos, aún desecados e insolados, los convierte en potenciales fuentes de infección, aunque deben existir factores externos, que aún no conocemos bien, que influyen poderosamente en la persistencia de Cryptococcus en este ambiente. 
1. Bou-Gabal M, Atia M. Study of the role of pigeons in the dissemination of Cryptococcus neoformans in nature. Sabouraudia 1978; 16: 63-68.

2. Álvarez LD, Llanos MI, Molina CE. Cryptococcus neoformans en excretas de palomas del perímetro urbano de Cali. Colombia Med 1996; 27: 106-109.

3. Bauters TGM, Moerman M, Pini G, Vermeersch H, Nelis HJ. Colonization of a voice prosthesis by Cryptococcus neoformans. Med Mycol 2001; 39: 379-381.

4. Cafarchia $C$, Romito $D$, latta $R$, Camarda A, Montagna MT, Otranto D. Role of birds of prey as carriers and spreaders of Cryptococcus neoforman and other zoonotic yeasts. Med Mycol 2006; 44: 485-492.

5. Cafarchia C, Camarda A, Romito D, Campolo M, Quaglia N.C, Tullio D, Otranto D. Occurrence of yeasts in cloacae of migratory birds. Mycopathologia. 2006; 161: 229-234

6. Caicedo LD, Álvarez MI, Llanos CE, Molina D. Cryptococcus neoformans en
excretas de palomas del perímetro urbano de Cali. Colombia Med 1996; 27 : urbano de

7. Casadevall A, Perfect JR. Cryptococcus neoformans. Washington D.C., ASM Press, 1998

8. Castañón Olivares LR Arreguín Espinosa R, Ruiz Palacios y Santos G, López Martínez R. Frequency of Cryptococcus species and varieties in México and their comparison with some Latin American Countries. Rev Latinoam Microbiol 2000; 42: 35-40.

9. Colom Valiente MF, Alberdi M, Meseguer I, Torres-Rodríguez JM. Aislamiento de Cryptococcus neoformans en muestras de medio ambiente de Alicante. Rev. Iberoam. Micol. 1997; 14: 63-64.

10. Colom MF, Frasés S, Ferrer C, Jover A, Andreu M, Reus S, Sánchez M, Torres JM. First human cryptococcosis due to Cryptococcus neoformans variety gattii in Spain. J Clin Microbiol 2005; 43 3548-3550.

11. Criseo G, Bolignano MS, De Leo F Staib F. Evidence of canary droppings as an important reservoir of Cryptococcus neoformans. Zentralbl Bakteriol 1995; 282:244-54.

12. Davis CE. Criptococos. En: Braude Al, Davis CE y Fierer J, (Eds.) Microbiología Clínica, $1^{\mathrm{a}}$ ed. Junín-Buenos Aires, Editorial Médica Panamericana, 1984: 722-732.

13. Emmons CW. Saprophytic sources of Cryptococcus neoformans associated with the pigeon (Columba livia). Am J Hyg 1955; 62: 227-232.

14. Emmons CW. Prevalence of Cryptococcus neoformans in pigeon habitats. Publ Health Rep 1960; 75: 362-364.

15. Fessel WJ. Cryptococcal meningitis after unusual exposures to birds. N Engl

16. García-Hermoso D, Mathoulin-Pélissier $S$, DNA typing Ronin O, Dupont B, Dromer as a source of pathogenic Cryptococcus as a source of pathogenic Cryptococcus 1997; 35: 2683-2685.

17. García Rodríguez JA, Picazo JJ. Microbiología Médica. 1. Microbiología Médica General, $1^{a}$ ed. Madrid: Harcourt Brace, 1998: 663-667.

18. Gatti M, DiSilverio A, Cespa M, Mosca M. Primary unusual cutaneous cryptococcosis in an HIV former drug-abuser patient. Mycoses 1997; 40: 101-102.
19. Glunder $\mathrm{G}$. Infections of pigeons as a risk to the health of humans and animals. Dtsch Tierarztl Wochenschr 1989; 96 : 112-116.

20. Gugnani HC, Sandhu RS, Shome SK. Prevalence of Cryptococcus neoformans in avian excreta in India. Mykosen 1976; 19: 183-187.

21. Haag-Wackernagel D, Moch H. Health hazards posed by feral pigeons. J Infect 2004; 48: 307-313.

22. Hermoso de Mendoza M Miranda García A, Leon Vizcaíno L, Perea JA, Carranza J, Gazquez A Del Pino J. Criptococosis espontánea en palomo. Arch Zootec 1984; 33: 27-41.

23. Hermoso de Mendoza M, Miranda A, Perea AJ, Arenas A, Poveda JB, Carranza J, Leon L. Criptococosis en paloma I. Frecuencia de portadores en buche en el área urbana de Córdoba. Rev Iberoam Micol 1987; 4: 121-127.

24. Jen TM. Cryptococcus albidus isolated from pigeon excreta in Taiwan. Zhonghua Min Guo Wei Sheng Wu Ji Mian Yi Xue Za Zhi 1992; 25:270-5.

25. Jover A, Ferrer C, Morán A, Gutiérrez J, Colom MF. Criptococosis por Cryptococcus albidus en un paciente inmunocompetente. Rev. Iberoam. Micol. 2006; 23: S3.

26. Kelley DC, Mossier JE. Public health aspects of mycotic diseases. J Am Vet Med Ass 1977; 171: 1168-1170.

27. Khan ZU, Pal M, Randhawa HS, Sandhu RS. Carriage of Cryptococcus $\mathrm{J}$ Med Microbiol 1978; 11: 215-218.

28. Khosravi AR. Isolation of Cryptococcus neoformans from pigeon (Columbia livia) droppings in northern Iran. Mycopathologia 1997; 139: 93-95.

29. Kielstein P, Bocklisch $\mathrm{H}$, Hotzel $\mathrm{H}$, Schmalreck A, Otto B. Evidence of Cryptococcus neoformans in domestic and sports pigeons in Thyringia. Mycoses 2000; 43: 23-28.

30. Kielstein P, Hotzel $H$, Schmalreck A, Khaschabi D, Glawischnig W. Occurrence of Cryptococcus spp. in excreta of pigeons and pet birds. Mycoses 2000; 43: 7-15.

31. Kobayashi CC, Souza LK, Fernandes Ode F, Brito SC, Silva AC Sousa ED, Silva Mdo R. Characterization of Cryptococcus neoformans isolated from urban environmental sources in Goiania, Goias State, Brazil. Rev Inst Med Trop Sao Paulo 2005; 47: 203-207.

32. Kordossis T, Avlami A, Velegraki A, Stefanou I, Georgakopoulos G, Papalambrou C, Legakis N.J. First report of Cryptococcus laurentii meningitis and a fatal case of Cryptococcus albidus cryptococcaemia in AIDS patients. Med Mycol 1998; 36: 335-339.

33. Kwon-Chung K.J, Boekhout T, Fell JW, Díaz M. Proposal to conserve the name Cryptococcus gattii against $C$. hondurianus and $C$. bacillisporus (Basidiomycota, Hymenomycetes, Tremellomycetidae). Taxon 2002; 51 : 804-806.

34. Lagrou K, Van Eldere J, Keuleers S, Hagen F, Merckx R, Verhaegen J, Peetermans WE, Boekhout T. Zoonotic transmission of Cryptococcus neoformans from a magpie to an immunocompetent patient. J Intern Med 2005; 257: 385-388.

35. Lazera MS, Pires FDA, Camillo-Coura L, Nishikawa MM, Bezerra CCF, Trilles L. Natural habitat of Cryptococcus neoformans var. neoformans in decaying wood forming hollows in living trees.
$\mathrm{J}$ Med Vet Mycol 1996; 34: 127-131.
36. Ledford $\mathrm{H}$. Hungry fungi chomp on radiation. news.nature.com. 070521 . 2007.

37. Levitz SM: The ecology of Cryptococcus neoformans and the epidemiology of
Cryptococcosis. Rev Infect Dis 1991; 13: Cryptococcosi

38. Li A, Guo N, Wu S. A strain of urease negative Cryptococcus neoformans isolated from the environment in China. Chin Med Sci J 1993; 8: 52-54.

39. Littman LM, Borok R. Relation of the pigeon to cryptococcosis: Natural carrie state, heat resistance and survival of Cryptococcus neoformans. Mycopathol Mycol Appl 1968; 35: 922-933.

40. Mancianti F, Nardoni S, Ceccherelli R. Occurrence of yeasts in psittacines droppings from captive birds in Italy.

41. Mangiarottii AM, Caretta G, De Luca C, Piontelli E. Hongos aislados del plumaje y excrementos de gallinas en una industria avícola de Monferrato (Pavia-Italia). Boletín Micológico 1993; 8: 91-98.

42. Mattsson R, Haemig PD, Olsen B. Feral pigeons as carriers of Cryptococcus laurentii, Cryptococcus uniguttulatus and Debaryomyces hansenii. Med Mycol 1999; 37:367-9.

43. Medleau L, Barsanti JA. Criptococosis. En: Green CE (Ed.) Enfermedades infecciosas. Perros y gatos. México, McGraw-Hill Interamericana 1993; 723-731.

44. Micalizzi C, Persi A, Parodi A. Primary cutaneous cryptococcosis in an immunocompetent pigeon keeper.
Clin Exp Dermatol 1997; 22: 195-197.

45. Nosanchuk JD, Shoham S, Fries BC, Shapiro DS, Levitz SM, Casadevall A. Evidence of zoonotic transmission of Cryptococcus neoformans from a pet cockatoo to an immunocompromised patient. Ann Intern Med 2000; 132 205-208.

46. Ordóñez N, Castañeda E. Serotipificación de aislamientos clínicos y del medio ambiente de Cryptococcus neoformans en Colombia Biomédica 1994; 14 131-39.

47. Pal M, Mehrotra BS. Studies on the isolation of Cryptococcus neoformans from fruits and vegetables. Mykosen 1984; 28: 200-205.

48. Pal M. Cryptococcus neoformans var. neoformans and munia birds. Mycoses 1989; 32: 250-252.

49. Pal M. First report of isolation of Cryptococcus neoformans var. neoformans from avian excreta in Kathmandu, Nepal. Rev Iberoam Micol 1997; 14: 181-183.

50. Passoni LF, Wanke B, Nishikawa MM, Lazera MS. Cryptococcus neoformans isolated from human dwellings in Rio de Janeiro, Brazil: an analysis of the domestic environment of AIDS patients with and without cryptococcosis. Med Mycol 1998; 36: 305-311.

51. Pollock C. Fungal diseases of columbiformes and anseriformes. Veterinary Clin. North. Am Exot Anim Pract 2003; 6: 351-361.

52, Quin PJ, Markey BK, Carter ME, Donnelly WJ, Leonard FC. Veterinary Microbiology and Microbial Disease, $1^{\mathrm{a}}$ ed. Oxford, Blackwell-Science 2002: 235-237.

53. Refai M, Taha M, Selim SA, Elshabourii F, Yousseff $\mathrm{HH}$. Isolation of Cryptococcus neoformans, Candida albicans and other yeasts from pigeon droppings in Egypt. Sabouraudia 1983; 21: 163-165. 
54. Rosario I, Acosta B, Déniz S, Padilla D, Soro G. Presencia de Cryptococcus spp en buche y heces de palomas mensajeras, y en cloacas (Columba livia) en la isla de Gran Canaria (España).

55. Rosario I, Hermoso de Mendoza M, Deniz S, Soro G, Alamo I, Acosta B. Isolation of Cryptococcus species including C. neoformans from cloaca of

56. Rubio C. Micosis subcutáneas y sistémicas. En: García Rodríguez JA y Picazo JJ, (Eds) Microbiología Médica. 1. Microbiología Médica General, $1^{\text {a }}$ ed.
Madrid, Harcourt Brace 1998: 663-667.

57. Ruiz A, Fromtling RA, Bulmer GS. Distribution of Cryptococcus neoformans in a natural site. Infect Immun 1981; 31: 560-563.

58. Schwarz J, Kauffman CA. Occupational hazards from deep mycoses. Arch Dermatol 1977; 113: 1270-1275.

59. Shrestha RK, Stoller JK, Honari G Procop GW, Gordon SM. Pneumonia due to Cryptococcus neoformans in a patien receiving infliximab: possible zoonotic transmission from a pet cockati Respir Care 2004; 49: 606-608.

60. Sorrell TC, Ellis DH. Ecology of Cryptococcus neoformans. Rev Iberoam Micol 1997; 14: 42-43.
61. Staib F, Schulz-Dieterich J. Cryptococcus neoformans in fecal matter of birds kept in cages - control of Cryptococcus neoformans habitats. Zbl Bakt Hyg I Abt Orig B 1984; 174: 179-186.

62. Stenderup J, Flensted K, Jorgensen C, Sorensen AH, Hansen NC, Siersted HC. Occurrence of the yeast, Cryptococcus neoformans, in pigeon droppings. Ugeskr Laeger 1989; 151: 2974-2975.

63. Swinne-Desgain D. Cryptococcus neoformans of saprophytic origin
Sabouraudia 1975; 13: 303-308.

64. Swinne-Desgain D. Cryptococcus neoformans in the crops of pigeons following its experimental administration. Sabouraudia 1976; 14: 313-317.

65. Tampieri MP. Mycetes and urban areas. Parassitologia 2006; 48: 121-124.

66. Tintelnot $\mathrm{K}$, Losert $\mathrm{H}$. Isolation of Cryptococcus adeliensis from clinical samples and the environment in 1007.

67. Tokojima M, Ihi T, Kyoraku Y, Hiratsuka T, Matsumoto K, Matsumoto N, Katoh S, Mukae H, Matsukura S. A case of bird fanciers' disease caused by parrot droppings. Nihon. Kokyuki Gakkai Zasshi 2001; 39: 739-743.
68. Vidotto V, Gallo MG. Study on the presence of yeasts in the feces of the rock pigeon (Columba livia) from rural areas. Parassitologia 1985; 27: 313-320.

69. Wegener HH, Staib F. Fatal cryptococcosis in a bird fancier. A clinical case report on pathology, diagnosis and epidemiology of cryptococcosis. Zentralbl Bakteriol Mikrobiol Hyg 1983; 256: 231-238.

70. Yamamoto $\mathrm{Y}$, Kohno S, Koga $\mathrm{H}$ Kakeya H, Tomono K, Kaku M, Yamazaki T, Arisawa M, Hara K. Ramdom Amplified Polymorphic DNA Analysis of clinically and environmental isolated Cryptococcus neoformans in Nagasak. $\mathrm{J}$ Clin Microbiol 1995; 33: 3328-3332.

71. Yilmaz A, Goral G, Helvaci S, Kilicturgay K, Gokirmak F, Tore O, Gedikoglu S. Distribution of Cryptococcus neoformans in pigeon feces. Mikrobiyol Bul 1989; 23 : in pigeon

72. Yimtubezenash WA, Leykun J, Dawit A. Isolation and characterization of Cryptococcus neoformans from environmental sources in Ethiopia. Ethiop J Health Dev 2001; 15: 45-50. 